\title{
Electrodeposición de recubrimientos de fosfato de calcio sobre titanio
}

\author{
E. Vidal ${ }^{1,2}$, E. Rupérez ${ }^{1,2}$, D. Rodríguez ${ }^{1,2}$ \\ I Biomaterials, Biomechanics and Tissue Engineering Group, Department of Materials Science and \\ Metallurgical Engineering, Universitat Politècnica de Catalunya (UPC) \\ 2 Center for Research in nanoEngineering, UPC
}

\begin{abstract}
Resumen
El titanio y sus aleaciones son biomateriales usados frecuentemente en implantes y prótesis. Sin embargo, estos biomateriales metálicos tienen limitaciones relacionadas con la bioactividad.

Una vía para aumentar la bioactividad del titanio es añadir un recubrimiento de fosfato de calcio (CaP) a la superficie mediante deposición electroquímica. Esta técnica tiene como ventajas la baja temperatura del proceso y un buen control del espesor y la composición química de la capa depositada. Durante el proceso, sin embargo, se producen burbujas de hidrógeno en las proximidades del cátodo, que dificultan la obtención de capas uniformes.

El presente estudio propone usar corriente pulsada para la electrodeposición como mecanismo para reducir la producción de burbujas de hidrógeno y mejorar el equilibrio de la concentración de iones en la solución, permitiendo la formación de capas de fosfato de calcio más uniformes.
\end{abstract}

Palabras clave: Titanio, electrodeposición, fosfato de calcio

\begin{abstract}
Titanium and its alloys are biomaterials commonly used in implants and prostheses. However, these metallic biomaterials have limited bioactivity.

One way to increase the bioactivity of titanium is to add a coating of calcium phosphate (CaP) to the surface by electrochemical deposition. This technique has as advantages the low temperature of the process and a good control of the thickness and chemical composition of the deposited layer. During the process, however, hydrogen bubbles may occur near the cathode, which makes it difficult to obtain uniform layers.

The present study proposes using pulsed current for electrodeposition as a mechanism to reduce the production of hydrogen bubbles and improve the equilibrium of the concentration of ions in the solution, allowing the formation of more uniform layers of calcium phosphate.
\end{abstract}

Keywords: Titanium, electrodeposition, calcium phosphate

Correspondencia:

Elia Vidal.

E-mail: elia.vidal@upc.edu

DOI: $10.5821 /$ sibb.26.1.8768 


\section{Introducción}

El titanio es un biomaterial con muy buena biocompatibilidad, en parte debida a su elevada resistencia a la corrosión en el medio fisiológico. El titanio presenta una buena resistencia a la corrosión debido a la gran reactividad que tiene con el oxígeno formando una capa de pasivación estable. La reactividad del titanio no da lugar a productos tóxicos, por lo que es ampliamente utilizado en aplicaciones biomédicas. Aunque biocompatible, el titanio no es bioactivo, lo que significa que no es capaz de inducir una actividad biológica específica [1].

Existen diversas alternativas para mejorar la bioactividad de un biomaterial metálico como el titanio, como por ejemplo tratamientos químicos que formen titanato en la superficie. Otros procesos se basan en la formación de un recubrimiento bioactivo (normalmente de fosfato de calcio) sobre el titanio, por proyección (plasma-spray), deposición sol-gel, o mediante laser-cladding, entre otras posibilidades. Entre estos procesos de recubrimiento, la deposición por pulverización de plasma es la única técnica utilizada comercialmente para recubrir prótesis, pero presenta ciertos inconvenientes, como la falta de uniformidad, el control limitado de la composición de la capa y la posible delaminación, debido a la altísima temperatura de procesado [2].

Otra vía para añadir un recubrimiento de fosfato de calcio a la superficie del titanio es mediante deposición electroquímica. Esta técnica tiene como ventajas la baja temperatura del proceso y un buen control del espesor y la composición química de la capa depositada. Durante el proceso, sin embargo, se producen burbujas de hidrógeno en las proximidades del cátodo, que dificultan la obtención de capas uniformes [3].

El presente estudio propone usar corriente pulsada para la electrodeposición como mecanismo para reducir la producción de burbujas de hidrógeno y mejorar la uniformidad de la concentración de iones en la solución, permitiendo la formación de capas de fosfato de calcio más uniformes.

\section{Materiales y Métodos}

Se prepararon muestras de titanio puro grado 2 de $10 \mathrm{~mm}$ de diámetro y $2 \mathrm{~mm}$ de espesor a partir de barras de titanio del mismo diámetro (Zapp Precision Metals GmbH, Alemania). Los discos fueron desbastados con discos de pulir de carburo de silicio desde 320 hasta 4000 grit (Buehler, EEUU) y finalmente pulidos con acabado espejo mediante sílice coloidal de 0,05um de diámetro.

Las muestras pulidas se limpiaron en un baño de ultrasonidos con soluciones de acetona, etanol y agua ultrapura. Antes de la electrodeposición, las muestras se trataron en una solución de $\mathrm{NaOH} 5 \mathrm{M}$ (Sigma Aldrich, EEUU) durante 24 horas a $60^{\circ} \mathrm{C}$, se enjuagaron con agua ultrapura y se secaron, almacenándose en un desecador.

La electrodeposición se realizó sobre las muestras de titanio con un equipo Parstat 2273 (Princeton Applied Research, EEUU) en régimen catódico. Se utilizó un electrodo de platino como ánodo. El electrolito se preparó mezclando $0,04 \mathrm{M} \mathrm{Ca}\left(\mathrm{NO}_{3}\right)_{2} \cdot 4 \mathrm{H}_{2} \mathrm{O}$ y $0,02 \mathrm{M}$ $\mathrm{NH}_{4} \mathrm{H}_{2} \mathrm{PO}_{4}$ (Sigma Aldrich). Las muestras se procesaron en el electrolito indicado durante 30 minutos a una temperatura de $40^{\circ} \mathrm{C}$ y una densidad de corriente de $2 \mathrm{~mA} / \mathrm{cm}^{2}$. Se compararon dos condiciones de corriente en el tratamiento (no pulsada / pulsada con pulso unipolar o bipolar, 300 ciclos de 4 segundos, figura 1).

Las propiedades fisicoquímicas del recubrimiento se evaluaron mediante diversas técnicas. La morfología y química superficial se estudió con microscopía electrónica de barrido complementada con sonda EDS (SEM Carl Zeiss NTS GmbH, Alemania). La microestructura del fosfato de calcio del recubrimiento fue analizada mediante difracción de rayos $\mathrm{X}(\mathrm{DRX})$ utilizando una radiación de $\mathrm{K} \alpha$ de $\mathrm{Cu}$ monocromática (Bruker D8 Advance Instrument, Alemania) a una velocidad de escaneo de $1 \%$, en el rango $2 \theta$ de 4 a $45^{\circ}$. La rugosidad del recubrimiento fue caracterizada con un equipo de interferometría de luz blanca WYKO NT9300 (Veeco Instruments, EEUU). 


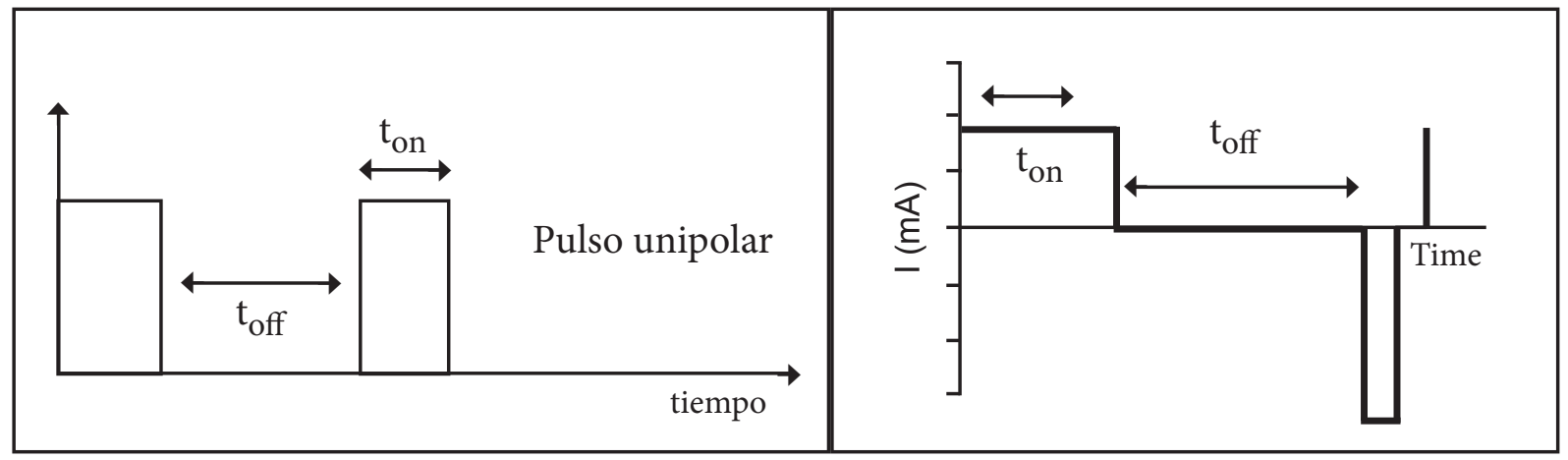

Figura 1. Esquema de electrodeposición en pulso unipolar (izquierda) y bipolar (derecha).

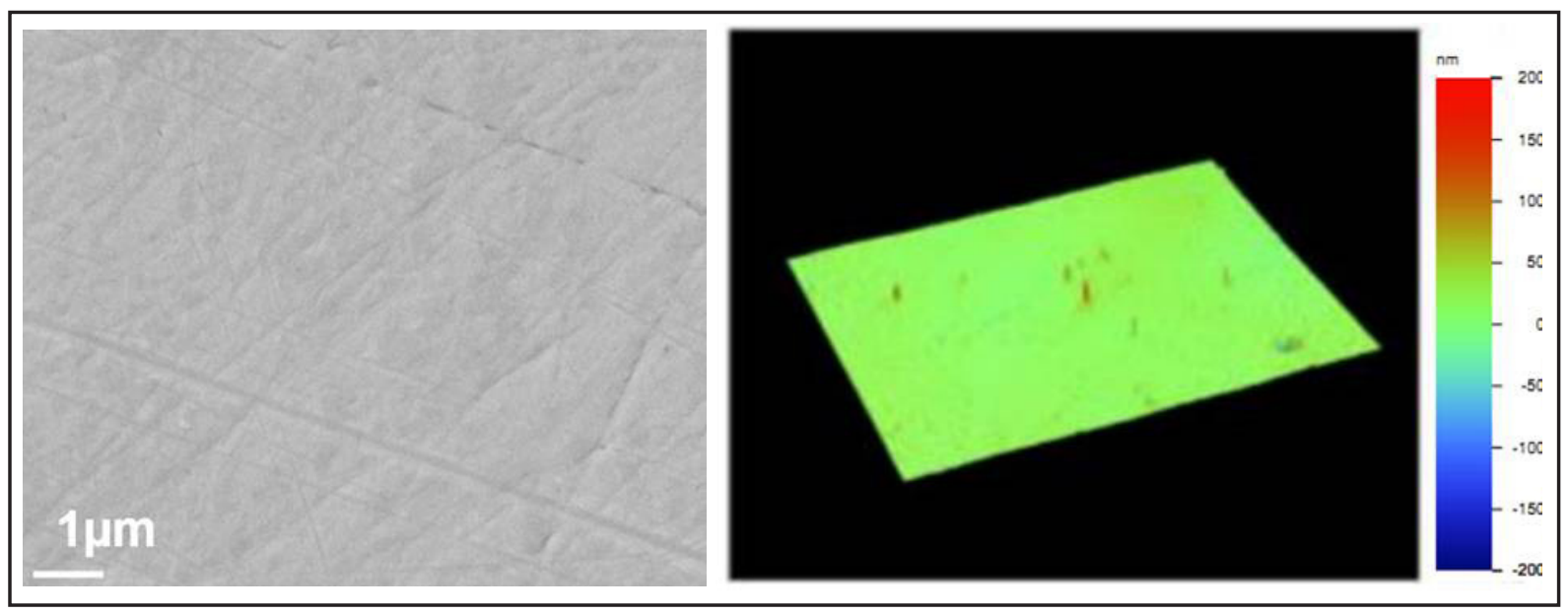

Figura 2. Imagen de microscopía electrónica de una muestra de titanio pulida, y reconstrucción de la topografía superficial mediante interferometría de luz blanca.
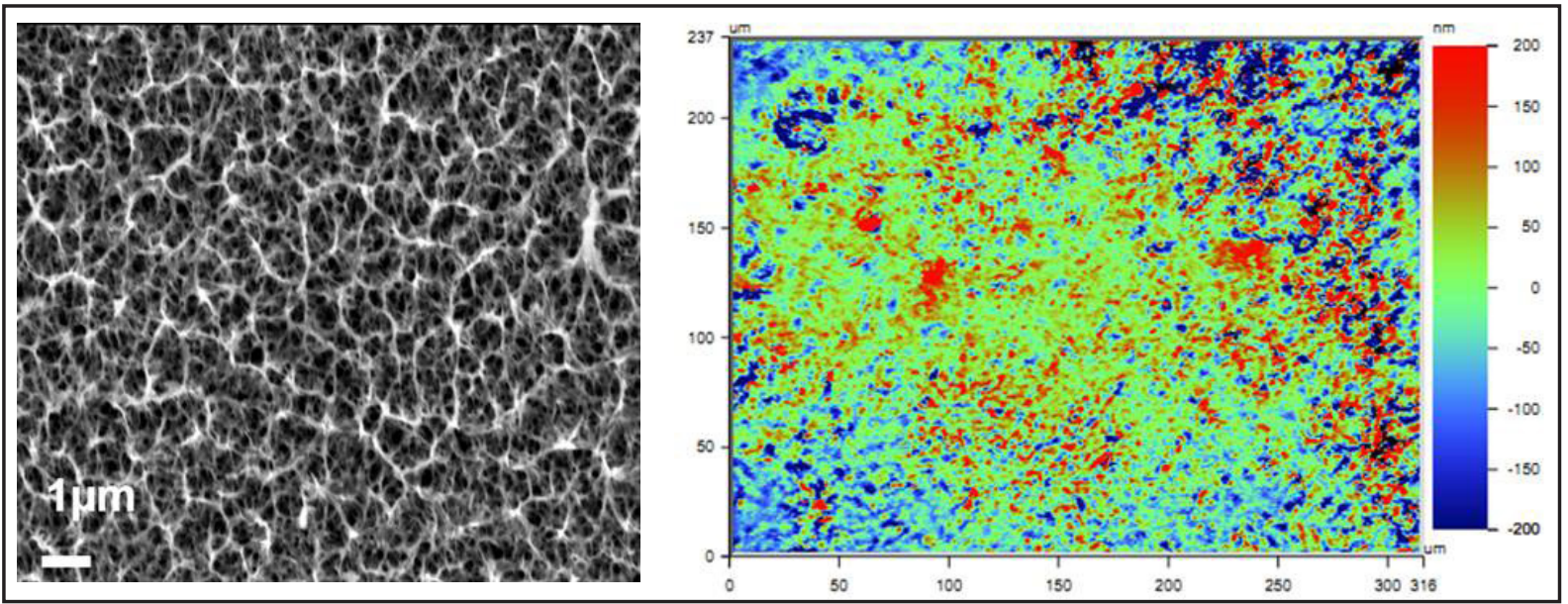

Figura 3. Imagen de microscopía electrónica de una muestra de titanio tratada con $\mathrm{NaOH}$, y reconstrucción de la topografía superficial mediante interferometría de luz blanca. 


\section{Resultados y Discusión}

Las muestras pulidas de titanio presentaron un acabado muy liso, como muestra la imagen de microscopio electrónico en la figura 2. Las medidas de rugosidad superficial con interferometría de luz blanca dieron una rugosidad media Ra de 12,6nm. Este acabado superficial permite evaluar los efectos de los tratamientos posteriores sin interferencias debidas al estado superficial previo.

Las muestras de titanio tratadas con una solución de $\mathrm{NaOH} 5 \mathrm{M}$ mostraron una superficie atacada con pérdida del pulido superficial (Figura 3). La rugosidad superficial Ra de las muestras tras el tratamiento es de 74,6nm. Esta evolución en la morfología superficial se debe al efecto corrosivo del $\mathrm{NaOH}$ en el titanio a temperaturas elevadas, y es empleado en algunas aplicaciones para incrementar la rugosidad superficial del titanio para aplicaciones biomédicas [4].

Tras el tratamiento electroquímico con corriente continua a $2 \mathrm{~mA} / \mathrm{cm}^{2}$, las muestras mostraban en la superficie el efecto de las burbujas de hidrógeno en el recubrimiento de fosfato de calcio formado sobre el titanio (figura 4) $[5]$.

La presencia de los defectos debidos a las burbujas de hidrógeno incrementa la rugosidad

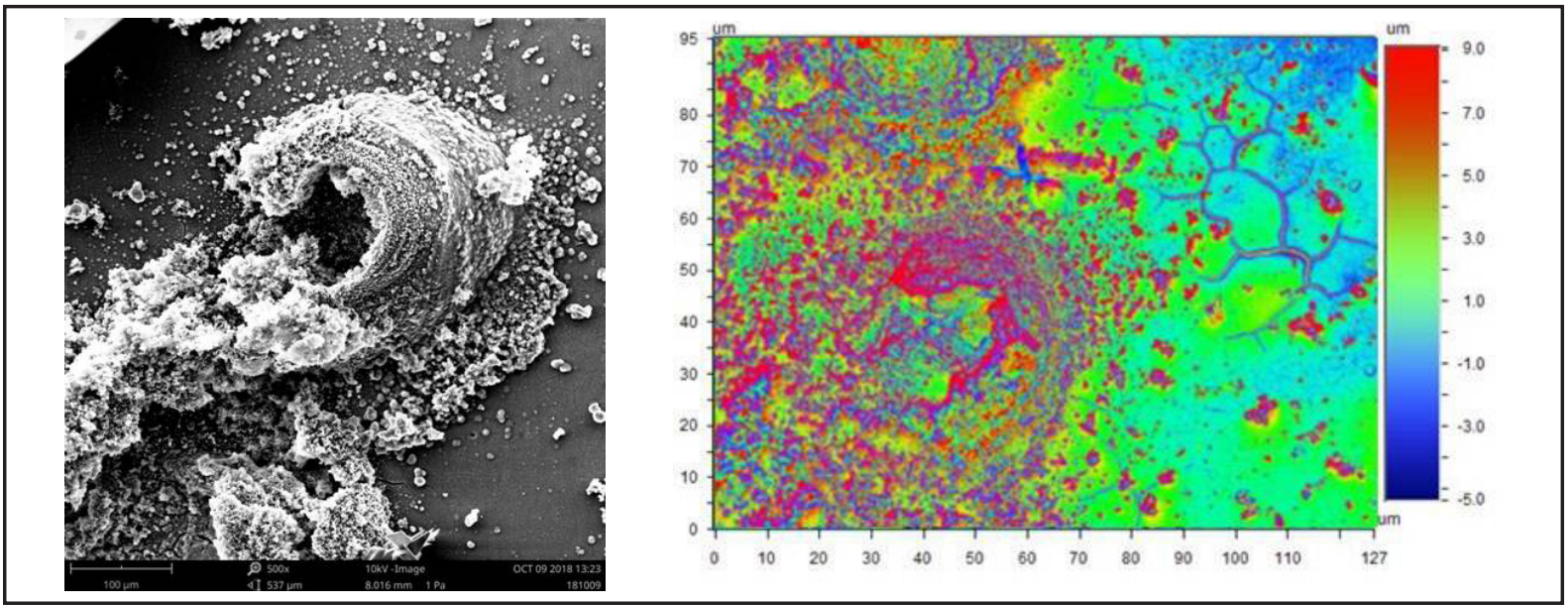

Figura 4. Imagen de microscopía electrónica de una muestra de titanio tras un tratamiento con corriente continua, y reconstrucción de la topografía superficial mediante interferometría de luz blanca.

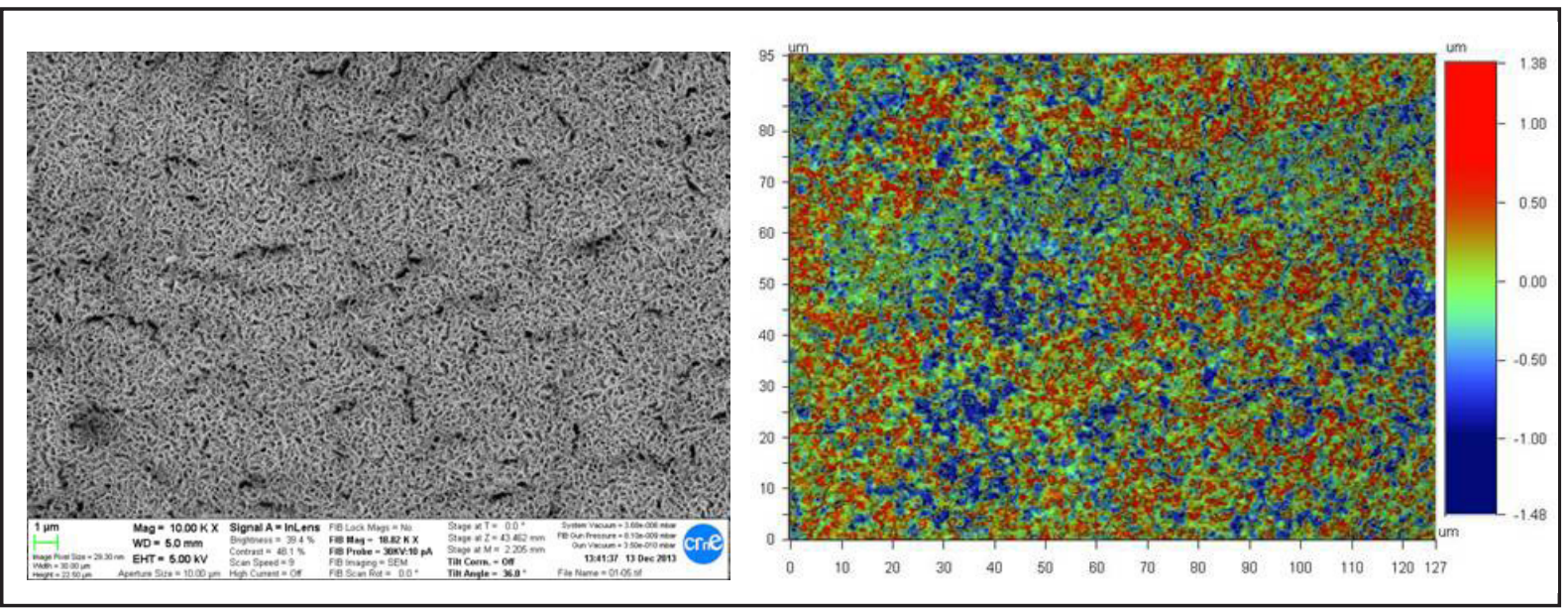

Figura 5. Imagen de microscopía electrónica de una muestra de titanio tras un tratamiento unipolar, y reconstrucción de la topografía superficial mediante interferometría de luz blanca. 
superficial a $1,80 \mu \mathrm{m}$. El valor de rugosidad incrementado no es un problema para la aplicación de este tratamiento, pero la presencia de los defectos debidos a las burbujas debilita la integridad estructural de la capa y hace más probable que se separe del sustrato [6]

En el caso de las muestras tratadas en régimen unipolar, las superficies presentaban una morfología compleja, con poros en la superficie (figura 5). Con este tratamiento, sin embargo, no se detectaron estructuras circulares como las que se habían observado en las muestras tratadas con corriente continua. La rugosidad superficial es de $0,32 \mu \mathrm{m}$, un valor bajo, como era de esperar al observar la morfología en microscopía electrónica.
Al examinar las superficies tratadas con más detalle, se observó la presencia de una morfología de tipo placas, con presencia de defectos en la continuidad de la capa (figura 6).

En el caso de los tratamientos electroquímicos en régimen bipolar, las superficies tratadas también presentaban superficies de morfología compleja, pero a diferencia de las muestras tratadas en régimen unipolar no se observaron poros o defectos en la superficie (figura 7). La rugosidad superficial es de 1,56 $\mu \mathrm{m}$, mucho mayor que la rugosidad en las muestras tratadas en régimen unipolar.

Al examinar la superficie tratada en régimen bipolar al microscopio electrónico con mayor resolución, se observó una morfolo-

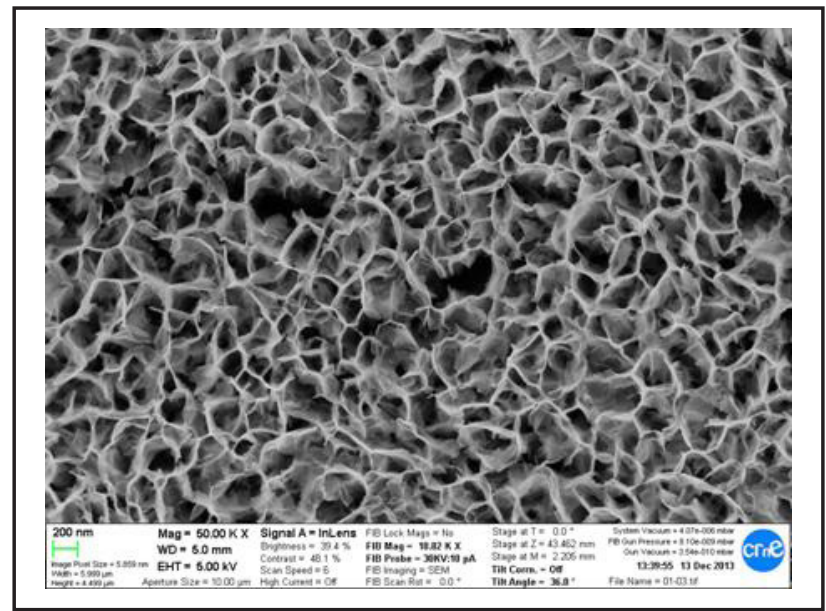

Figura 6. Detalle de la superficie de una muestra de titanio tras un tratamiento electroquímico unipolar.

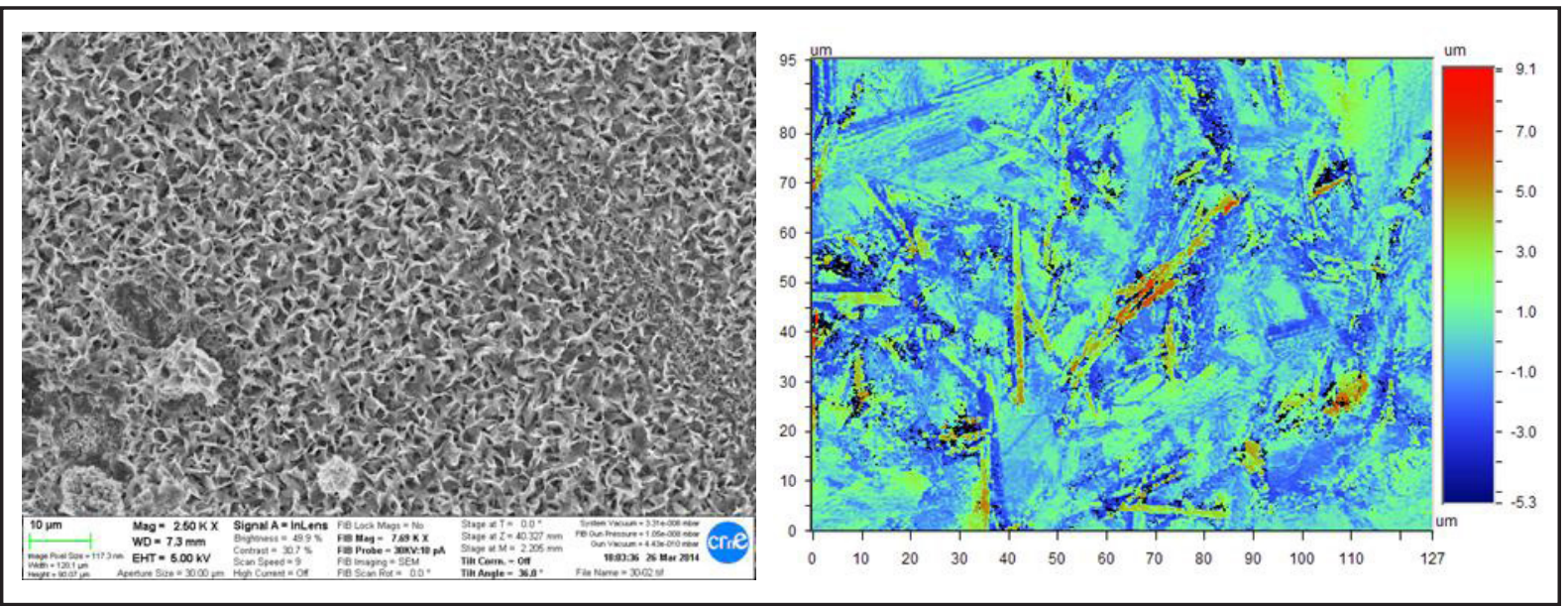

Figura 7. Imagen de microscopía electrónica de una muestra de titanio tras un tratamiento con corriente bipolar, y reconstrucción de la topografía superficial mediante interferometría de luz blanca. 
gía de placas similar a la obtenida en régimen unipolar, pero mucho más homogénea, y con mayores tamaños de placas (Figura 8).

Las observaciones en microscopía electrónica permiten comprobar que el uso de corriente pulsada permite obtener capas de fosfato de calcio más uniformes. La interrupción de la corriente no permite la formación de grandes burbujas, pero como se observa en las muestras tratadas en régimen unipolar, no desaloja las burbujas de la superficie, generando defectos. Sólo las muestras tratadas con un régimen bipolar consiguen desalojar las burbujas de la superficie, facilitando la formación de la capa de fosfato de calcio en la superficie de titanio al no interferir las burbujas de hidrogeno [7].

El análisis de las muestras tratadas con corriente pulsada mediante difracción de rayos $\mathrm{X}$ muestra en el espectro obtenido picos cuya indexación corresponde la presencia de fases cristalinas de fosfato de calcio (figura 9).

En el caso de las muestras tratadas con corriente unipolar, se detectaron picos cuya indexación corresponde a la hidroxiapatita, brushita y fosfato octacálcico (OCP). Esto puede ser debido a un aumento de la concentración de iones hidroxilo en el electrolito que promueve la precipitación de OCP. Estas fases de fosfato de calcio podrían influir en el cre-

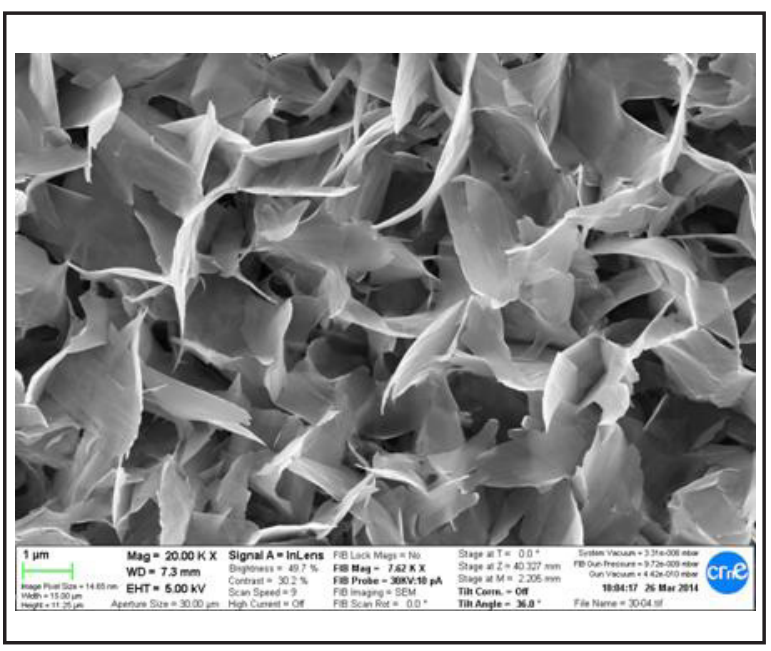

cimiento óseo, ya que se conocen como precursores de la formación de HA. El espectro de DRX de las muestras tratadas en régimen bipolar muestra picos de brushita y OCP, pero no de hidroxiapatita (figura 9).

En ambos casos, el espectro muestra una señal poco intensa de titanio metálico fase $\alpha$, hecho que sugiere que la capa de fosfato de calcio tiene un espesor micrométrico, estimado en 5-10 $\mu \mathrm{m}$.

\section{Conclusiones}

Se crearon con éxito recubrimientos de fosfato de calcio en la superficie de muestras de titanio mediante un proceso electroquímico. Se ha conseguido mejorar la calidad de la capa obtenida ajustando el pulsado de la corriente aplicada al sistema para reducir la formación de burbujas en la superficie a tratar.

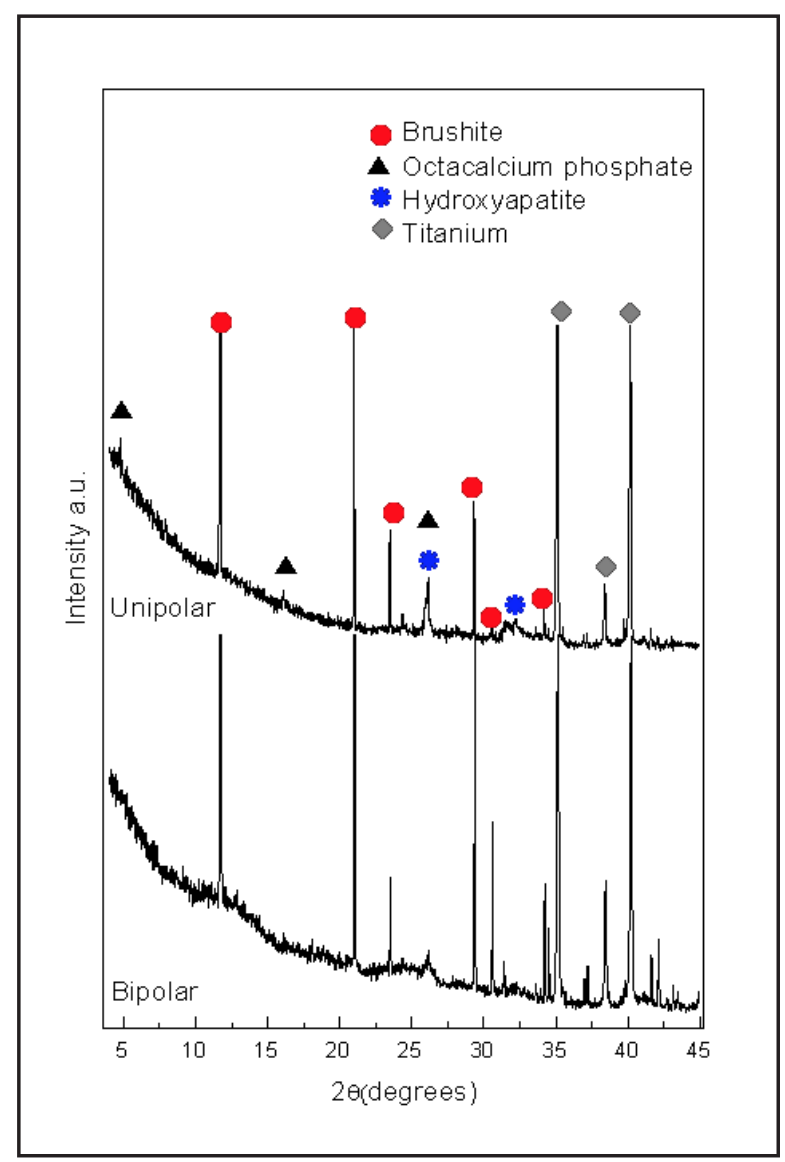

Figura 8. (Izquierda) Detalle de la superficie de una muestra de titanio tras un tratamiento electroquímico bipolar.

Figura 9. (Derecha) Espectro de DRX de una muestra de titanio tras un tratamiento electroquímico pulsado. 


\section{Agradecimientos}

El presente estudio fue apoyado por la Agencia Catalana d'Administració de Beques Universitàries $\mathrm{i}$ de Recerca (AGAUR FI-DRG). Los autores reconocen al Gobierno español para el apoyo financiero a través del proyecto MAT2015-67183-R, co-financiado por la Unión Europea a través de los Fondos Europeos de Desarrollo Regional.

\section{Referencias}

1 Scharnweber $D$, Flössel $M$, Born $R$, Worch H. Adjusting the chlorhexidine content of calcium phosphate coatings by electrochemically assisted co-deposition from aqueous solutions. J Mater Sci Mater Med 2007;18:391-397.

2 García-Sanz FJ, Mayor MB, Arias JL, Pou J, León B, Pérez-Amor M. Hydroxyapatite coatings: a comparative study between plasma-spray and pulsed laser deposition techniques, J Mater Sci Mater Med 1997;8:861-865.
3 Djošić MS, Panić V, Stojanović J, Mitrić M, Miskovic-Stankovic VB. The effect of applied current density on the surface morphology of deposited calcium phosphate coatings on titanium, Colloids Surf. A Physicochem Eng Asp. 2012;400:36-43.

4 Fujibayashi S, Neo M, Kim HM, Kokubo T, Nakamura T. Osteoinduction of porous bioactive titanium metal. Biomaterials. 2004;25(3):443-450.

5 Vidal E, Buxadera-Palomero J, Pierre C, Manero JM, Ginebra MP, Cazalbou S, Combes C, Rupérez E, Rodríguez D. Single-step pulsed electrodeposition of calcium phosphate coatings on titanium for drug delivery. Surf Coat Techol. 2019;358:266-275.

6 Gopi D, Indira J, Kavitha L. A comparative study on the direct and pulsed current electrodeposition of hydroxyapatite coatings on surgical grade stainless steel, Surf Coat Technol. 2012;206:2859-2869.

7 Chandrasekar MS, Pushpavanam M. Pulse and pulse reverse plating - conceptual, advantages and applications. Electrochim Acta. 2008;53:3313-3322. 